\title{
Development of Residual Stresses During Laser Cladding
}

\author{
A. Narayanan ${ }^{a}$, M. Mostafavi ${ }^{b}$, M. Pavier ${ }^{c}$ and M. Peel ${ }^{d^{*}}$ \\ ${ }^{1}$ University of Bristol, Department of Mechanical Engineering, Queen's Building, University \\ Walk, Bristol, BS8 1TR, United Kingdom \\ aa.narayanan@bristol.ac.uk, ${ }^{b}$ m.mostafavi@bristol.ac.uk, cmartyn.pavier@bristol.ac.uk, \\ matthew.peel@bristol.ac.uk
}

\section{Keywords: Cladding, Synchrotron X-Ray Diffraction, Stress-Measurement, Rail}

\begin{abstract}
Laser cladding rail steel with a hard-wearing martensitic stainless-steel coating is a possible technique for improving the track durability of rail networks. However, the cladding process induces significant residual stresses in the clad material, due to the thermal mismatch between the two materials and the shape changes during the martensitic phase transformation. Predictions of the residual stress remain poorly verified as the process is complex and measurements made on final clad parts can be influenced by multiple parameters.

A cladded and heat-treated rail section was subject to sequential laser-pulses representative of the actual cladding process. The thermal cycle of these pulses is much simpler than real clads, easing the task of validating the component parts of simulations. Synchrotron X-ray diffraction was used to determine the phase selective residual stresses around the heated region before and after each pulse. In this manner it was possible to determine the change in stress due to a pulse and the degree of relaxation that is possible due to a neighbouring thermal cycle.
\end{abstract}

\section{Introduction}

A typical piece of rail sees many train wheels passing over it each day. The contact forces involved are high, and involve a mixture of dynamic and static loading, the combination of which can precipitate crack formation and wear towards the surface of the rail. To mitigate against this, it is possible to coat a section of generic rail steel (the substrate material) with a thin layer of a harder, more damage resistant alloy (the clad material) using laser cladding. This involves depositing a powdered form of the clad material onto the surface of the substrate and using a laser to melt it and form a coating. The process involves high, localized thermal gradients occurring over a short time-span and can therefore induce residual stresses in the clad-substrate system. Residual stresses interact with the applied loads to create more complex stress states, the result of which can cause damage at loads that would normally be considered to be within safe limits [1,2] and it is important to be able to understand and predict this interaction.

Martensitic stainless steel is a potential clad but stress development during cooling is complicated by the volumetric and shear strains that occur during the martensitic phase transformation. These can be difficult to account for in simulations [3-5], particularly when the transient temperatures are themselves hard to model. The thermal excursion has been simplified by using a single, tightly-controlled, laser pulse and then measured the residual stresses between pulses. This has been performed to provide a simple analogue of the heat input to the material while minimising the practical difficulties of powder deposition in an X-Ray beamline. If later finite element analysis (FEA) can predict the stress state accurately while incorporating effects of phase transformations, it can be extended for the full laser cladding simulations. Synchrotron Xray diffraction using 2D imaging provides excellent spatial resolution, far in excess of lab X-ray and neutron diffraction, and simultaneous measurement of multiple phases. 


\section{Materials and Methods}

Clad samples were cut from a previously clad rail, $250 \mathrm{~mm}$ in length, resulting in $2 \mathrm{~mm}$ thick slices with the dimensions shown in Figure 1. The clad had been applied by laser cladding in two layers to a depth of $2 \mathrm{~mm}$, before being ground down to provide a smooth surface finish with a clad approximately $1.2 \mathrm{~mm}$ thick. The clad consists of a martensitic stainless steel (hereafter referred to as MSS, and whose composition is detailed in Table 1), while the substrate was rail steel grade 260 (also known as UIC 900A) [6]. The use of pre-clad rail ensured the microstructure of the martensite was typical of laser-deposited material. Pre-existing residual stresses were relieved, so far as possible, by tempering the samples at $600^{\circ} \mathrm{C}$ for two hours.

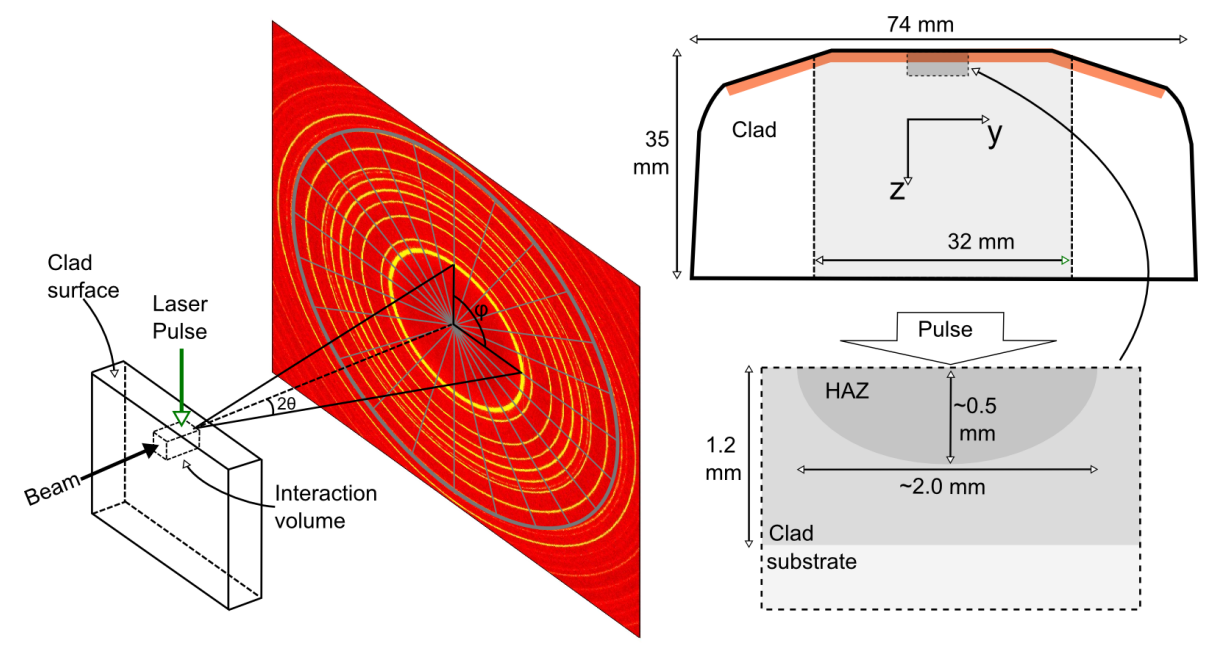

Figure 1. Illustrations of the sample and setup. The orientation of the sample (left) with respect the incoming beam and detector with the diffraction angle $2 \theta$ and azimuthal angle $\varphi$ defined. The sample (right) is cut from a larger rail and was positioned with the laser above it so that the hot zone lies within the clad. The nominal heat affected zone (HAZ) is shown but is only a guide.

Table 1 Composition of MSS clad material including main alloying elements

\begin{tabular}{|l|l|l|l|l|l|l|l|}
\hline Element & $\mathrm{C}$ & $\mathrm{Mn}$ & $\mathrm{Si}$ & $\mathrm{Cr}$ & $\mathrm{Ni}$ & $\mathrm{Mo}$ & $\mathrm{Fe}$ \\
\hline Wt \% & 0.04 & 0.8 & 0.6 & 13.0 & 4.1 & 0.5 & Balance \\
\hline
\end{tabular}

The experiment was performed by positioning a $500 \mathrm{~W}$ laser above the specimen and focusing it on the clad surface. The laser produced a pulse of set dimensions of $1.4 \mathrm{~mm}$ diameter with a set duration of $0.015 \mathrm{~s}$. This would theoretically generate an axisymmetric molten zone on the upper surface, inducing austenitisation to a greater depth followed by martensite formation during the rapid cool. The width of the hot zone was approximately equal to the thickness of the clad slice and about half the depth of the clad (Figure 1). The temperature of the rear surface (facing beam) was measured using a FLIR T650sc thermal imaging camera operating at $30 \mathrm{~Hz}$.

Stresses were measured using synchrotron X-rays on the ID31a beamline at the European Synchrotron Radiation Facility (ESRF). A monochromatic beam of wavelength $0.0173 \mathrm{~nm}$ was focussed onto the sample with a spot size of $40 \times 15 \mu \mathrm{m}$. The interaction volume covered the full thickness of the sample. Due to the wide laser spot size it is assumed the temperature is uniform throughout this volume. The detector was a Pilatus Cadmium-Tellurium (CdTe) 2D detector that allowed for individual photon counting, resulting in patterns with very low noise and high sensitivity to small phase fractions. Tilts were corrected using an image from a Ceria powder sample. Each image was integrated azimuthally into 36 patterns (See Figure 2). It is notable that blank regions between detector sections mean that some reflections are missing or distorted. The 
measurement was from the initial clad and $\alpha$ (i.e. martensite) dominates with only small amounts of retained $\gamma$ phases. The number of counts away from peaks (i.e. the background) is extremely low and even small peaks are easily resolved and fittable. Peaks were fitted using a pseudovoigt function, which was found to accurately match the peak shape. Fitting has been limited to nonoverlapping peaks i.e. ferritic (martensite): (200), (211), (220) and austenite: (220), (222), (311).
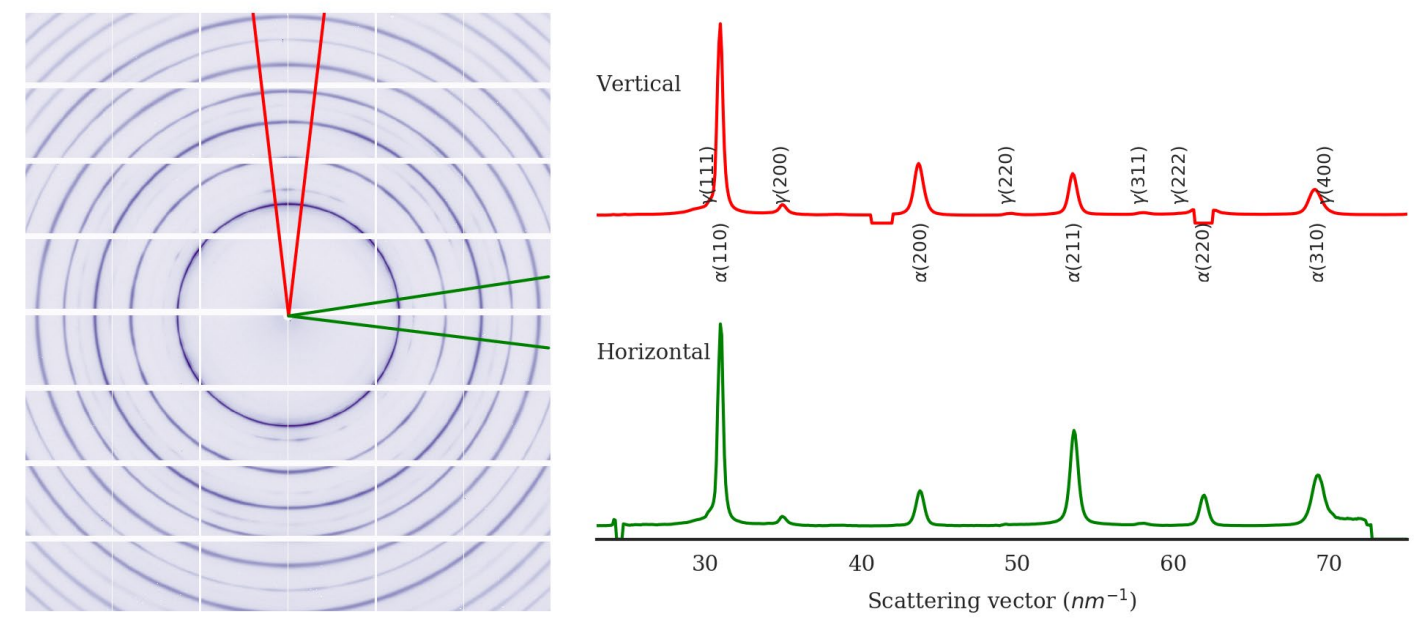

Figure 2. A sample image obtained using the Pilatus detector (left) and the patterns derived from the results (right) with the peaks families labelled. The patterns show the sqrt(I) to emphasise smaller peaks.

The sample had three pulses applied to it sequentially and separated laterally (centre-tocentre, y-direction) by $1 \mathrm{~mm}$. The purpose of this was to mimic the effect of creating a continuous clad. The residual stress field before and after each pulse was measured over a 4 x 1 mm region, entirely within the clad, with a grid spacing of around $100 \mu \mathrm{m}$ in the z-direction and $140 \mu \mathrm{m}$ in the $\mathrm{y}$-direction. The low diffraction angle $\left(<10^{\circ}\right)$ means the scattering vectors for all peaks lie close to the plane of the clad slice. The strain from the peak shift $\Delta q$ for any (hkl) peak is given by

$$
\varepsilon_{\phi}=\frac{\Delta q}{q_{0}^{h k l}}=p_{11} \sigma_{11}+p_{12} \sigma_{12}+p_{22} \sigma_{22}
$$

where $q_{0}^{h k l}$ is the peak position in the absence of a stress and $p_{i j}$ are the stress factors. These incorporate both the direction (i.e. azimuthal angle, $\varphi$ ) and the $(h k l)$ dependent diffraction elastic constants $\left(S_{1}, 1 / 2 S_{2}\right)$. Ignoring the slight out-of-plane component of the scattering vector, assuming alignment of the lab and sample coordinate systems, and setting $\varphi=0$ for the vertical detector element gives

$$
p_{i j}=\left\{\begin{array}{l}
\frac{1}{2} S_{2} h_{i}^{2}+S_{1} \text { if } i=j \\
2 \cdot \frac{1}{2} S_{2} h_{i} h_{j} \text { if } i \neq j
\end{array} \quad \text { where } h=\left[\begin{array}{c}
\cos \varphi \\
\sin \varphi
\end{array}\right]\right.
$$

The diffraction elastic constants were obtained using the Isodec software without texture [7]. For each measurement and each phase there are $3(h \mathrm{kl})$ per spectra and 36 spectra at different $\varphi$ angles. The stress components can be determined by solving the resulting set of linear equations. 


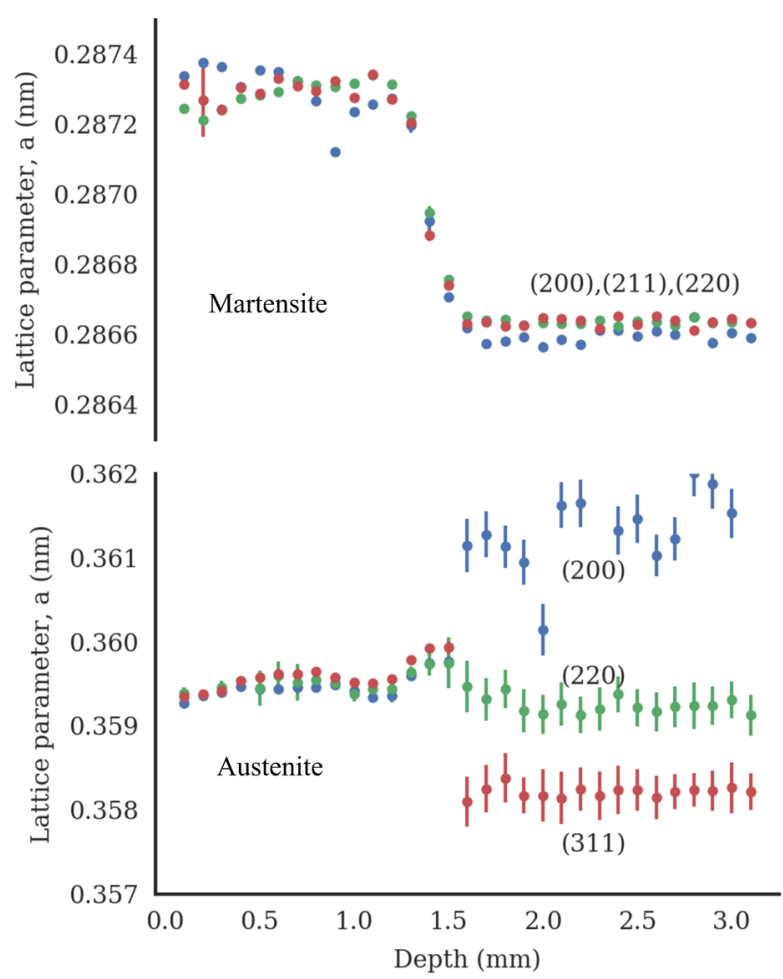

Figure 3 The lattice parameter of the martensite and austenite measured as a function of depth below the clad surface near to the edge of the sample. The clad finishes about 1.2. The low volume fraction of austenite in the substrate (>1.5 mm) makes values beyond this depth unreliable.

\section{Results}

Accurate values for $q_{0}^{h k l}$ were determined as a function of depth by measurements at the edge of the initial sample (Figure 3). The stress state is expected to be low and the location means most remaining stresses should be locally relieved. Since $q^{h k l}$ was broadly constant over the clad $(<1.2 \mathrm{~mm}) q_{0}^{h k l}$ was set to be the simple average for each $(h k l)$.

Plots of residual stress components in the z-direction and y-direction (normal and transverse in the original rail) are shown in Figure 4 for the martensite phase. The four maps show the initial stress state and after 1, 2 or 3 pulses, respectively. The initial sample has uniform compressive stress in both directions $(<-$ $200 \mathrm{MPa}$ ). The austenite is uniformly in tension in both directions ( $<200 \mathrm{MPa}$ ) except on the very upper layer where it is unstressed or slightly compressive.

A laser pulse results in a region of high tensile stress broadly corresponding to the material heated above the austenite transformation temperature, and hence reformed martensite upon cooling. The maximum stress in the z-direction occurs around $0.5 \mathrm{~mm}$ below the surface - this stress component tends to reduce towards the upper surface as expected from boundary conditions. This stress component (tensile in the martensite, compressive in the austenite) probably doesn't reach zero due to interphase stresses, which balance each other but do not have to be zero at the surface. In the y-direction, the highest tensile stresses are located closer to the upper surface but are of similar magnitude. Compressive stresses are seen at greater depths with the highest magnitude lying just below the tensile region. The residual stress in the austenite (Figure 5) is a mirror image of the martensite with compression in the hottest region and tensile stresses at greater depths. The austenite stress appears more hydrostatic in character with similar magnitudes in both directions. The measured tensile stress at around $0.5 \mathrm{~mm}$ depth is perhaps unrealistically high (700-900 $\mathrm{MPa}$ ) but then this retained austenite will be widely distributed between martensite laths, so high interphase stresses are likely.

Sequential pulses are remarkably consistent. The size and shape of the heat affected zone, and the magnitude of stress components within it, appears independent of the number of pulses and the new zone seems to overwrite the zone next to it. There is a modest tempering effect on the neighbouring pulses, which then appear smaller in extent and magnitude. This is clearer in line plots showing the stress components as a function of depth at the nominal position of each pulse in the final sample (Figure 6). The first and second pulses are almost identical in stress indicating that additional pulses, further away from the first pulse, have little additional tempering effect. The third pulse systematically shows larger magnitude stresses, although not in all locations. 


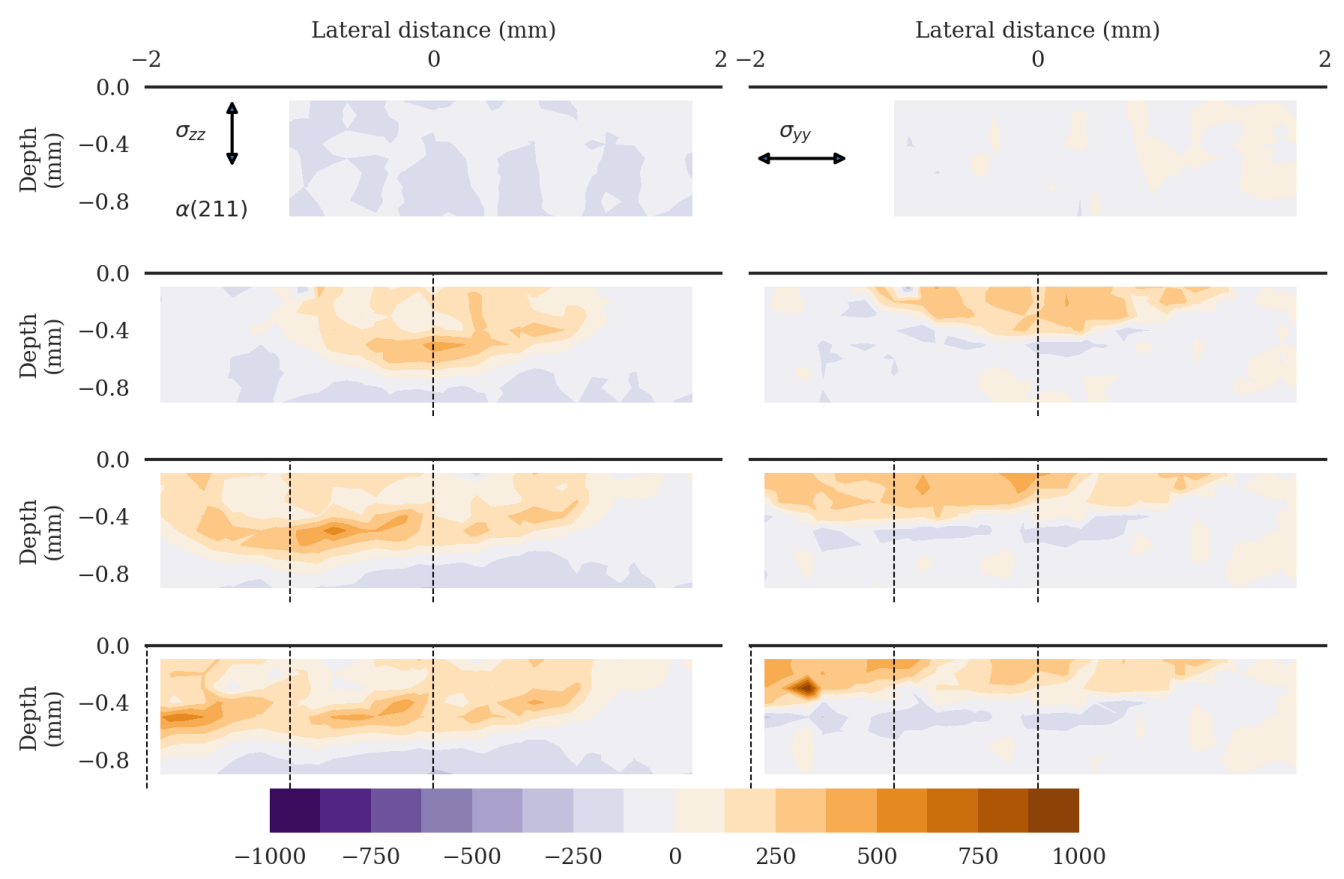

Figure 4. Plots of the $\mathrm{z}$ and y components of residual stress (MPa) in the martensite, as measured with the (211) reflection, for sequential laser pulses. The dotted lines show nominal pulse centres.

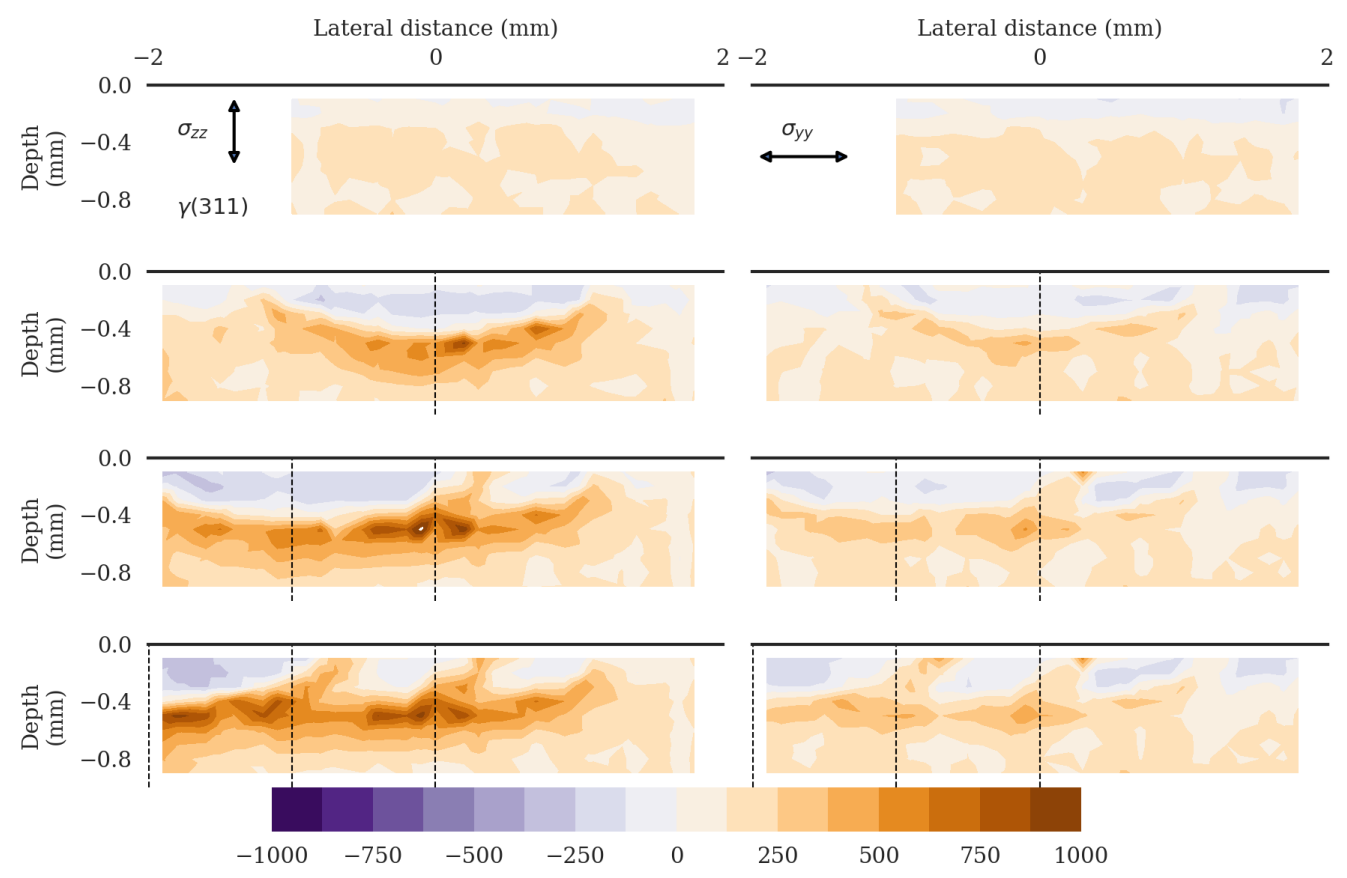

Figure 5. Plots of the $\mathrm{z}$ and $y$ components of residual stress (MPa) in the austenite, as measured with the (311) reflection, for sequential laser pulses. The dotted lines show nominal pulse centres.

\section{Conclusions}

An experiment was performed to investigate how laser welding changes the residual stress state. A series of three adjacent welds was made to simulate the effect of running a longer track of laser clad. The combination of excellent beam focussing, and a high-speed, low noise detector allows excellent spatial resolution and stress measurement, even in a minority phase. 


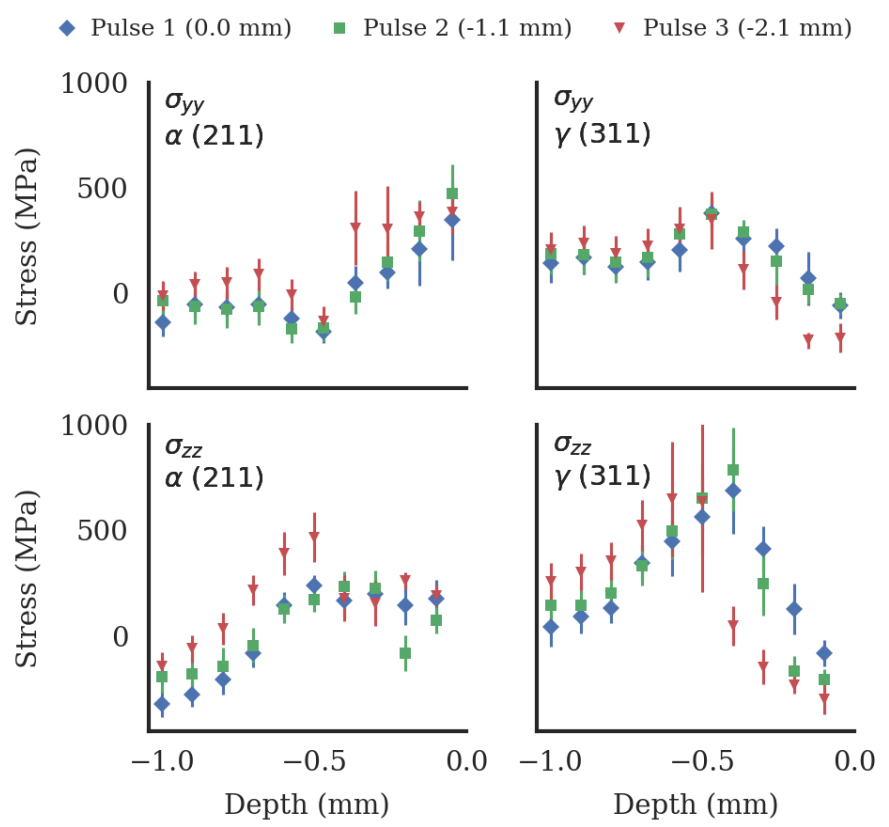

Figure 6. Stress profiles for two orthogonal stress components along the centreline of initial weld (same location of pulse 1); results include the effect of adjacent welds with stress plotted after pulse 1 (blue diamonds), pulse 2 (green squares and pulse 3 (red triangles) and error bars show 95\% confidence intervals.

Results have shown that forming a spot weld on the surface of a martensitic clad on UIC 900A substrate produces large tensile stress in the weld zone. This stress is uniaxial towards the surface but becomes biaxial at approximately $0.4 \mathrm{~mm}$ depth. The stress components exceed $600 \mathrm{MPa}$ in some regions but the location of maxima and minima is different for each component. The stress state is highly consistent and repeatable in both the ferritic and austenitic phases.

Overlaying multiple pulses starts to approximate a continuous clad more typical of genuine cladded samples. The data suggest that sequential pulses does cause slight tempering of prior adjacent welds. The effect is modest but exceeds the uncertainty in the measurements. Whether this effect is significant enough to need to be included in simulations remains to be seen.

\section{Acknowledgements}

This research was funded by EPSRC research grant EP/M023044/1, with equal contributions from EPSRC and the Rail Safety and Standards Board (RSSB). The authors would like to thank the Rail Safety and Standards Board (RSSB) for its support in conducting this research.

\section{References}

[1] A. Mirzaee-Sisan, C. E. Truman, D. J. Smith, and M. C. Smith, Eng. Fract. Mech. 74, 2864 (2007). https://doi.org/10.1016/j.engfracmech.2006.12.033

[2] G. A. Webster and A. N. Ezeilo, Int. J. Fatigue 23, Supplement 1, 375 (2001). https://doi.org/10.1016/S0142-1123(01)00133-5

[3] H. Dai, J. A. Francis, H. J. Stone, H. K. D. H. Bhadeshia, and P. J. Withers, Metall. Mater. Trans. A 39, 3070 (2008). https://doi.org/10.1007/s11661-008-9616-0

[4] J. Altenkirch, J. Gibmeier, V. Kostov, A. Kromm, T. Kannengiesser, S. Doyle, and A. Wanner, J. Strain Anal. Eng. Des. 46, 563 (2011). https://doi.org/10.1177/0309324711413190

[5] R. A. Ainsworth, J. K. Sharples, and S. D. Smith, J. Strain Anal. Eng. Des. 35, 307 (2000). https://doi.org/10.1243/0309324001514431

[6] J. W. Ringsberg, A. Skyttebol, and B. L. Josefson, Int. J. Fatigue 27, 702 (2005). https://doi.org/10.1016/j.ijfatigue.2004.10.006

[7] T. Gnäupel-Herold, J. Appl. Crystallogr. 45, 573 (2012). https://doi.org/10.1107/S0021889812014252 